\title{
Pavlovian conditioning in multiple contexts: Competition between contexts for comparator status
}

\author{
NICHOLAS J. GRAHAME, ROBERT C. BARNET, and RALPH R. MILLER \\ State University of New York, Binghamton, New York
}

\begin{abstract}
Water-deprived rats were used to investigate the effects of training a CS in more than one context on conditioned lick suppression. In each experiment, partial reinforcement of the CS was intermingled with unsignaled presentations of the US. In Experiment 1, subjects were either trained in one context alone, trained consecutively in two contexts (such that all training in one context occurred prior to any training in the second context), or trained alternately in two con. texts. Following training, the first context, the second context, or neither context was extinguished. Testing of the CS occurred in a third (neutral) context. To the extent that either training context became established as a comparator stimulus for the CS, the comparator hypothesis (Miller \& Matzel, 1988) predicts an increase in excitatory responding to the CS following extinction of that context. Subjects trained in a single context exhibited appreciable fear of the CS only when the CS's training context had been extinguished. Additionally, subjects trained consecutively in the two contexts showed increased fear of the CS following extinction of the second, but not the first training context (i.e., a recency effect). Subjects trained alternately in the two contexts showed no increased fear of the CS as a result of either context alone being extinguished. In Experiment 2, subjects trained alternately in two contexts showed increased fear of the CS only when both training contexts were extinguished, suggesting that both training contexts had become comparator stimuli. These data indicate that multiple training contexts can either compete or act synergistically in modulating responding to a Pavlovian trained CS as a function of the order of training in the different contexts.
\end{abstract}

The comparator hypothesis (Miller \& Matzel, 1988; Miller \& Schachtman, 1985; but see Miller, Hallam, \& Grahame, 1990 for limitations) is a response rule that posits that responding to a conditioned stimulus (CS) is directly related to the strength of the association between the CS and the unconditioned stimulus (US) and is inversely related to the strength of associations between the US and other cues (i.e., comparator stimuli) that were present during training of the CS and the US. Recent evidence indicates that comparator stimuli have modulatory effects that are specific to those CSs trained in their presence (Miller, Barnet, \& Grahame, 1992). Comparator stimuli may include training context cues, but are not limited to them (Hallam, Matzel, Sloat, \& Miller, 1990). The comparator hypothesis predicts that a CS will function as a conditioned excitor when its associative strength is large relative to its comparator stimuli, and will function as a conditioned inhibitor when its associative strength is small relative to its comparator stimuli. In other words, the as-

Support for this research was provided in part by National Institute of Mental Health Grant 33881 and the SUNY-Binghamton Center for Cognitive and Psycholinguistic Studies. Thanks are due to James Esposito for collecting the data, and to Steve Hallam for commenting on a preliminary version of the manuscript. Requests for reprints should be addressed to R. R. Miller, Department of Psychology, SUNYBinghamton, Binghamton, NY 13902-6000. sociative status of comparator stimuli modulates responding to a CS.

The comparator hypothesis posits that conditioned responding depends on three associations. The first is the conventional CS-US association. The second is a CS-comparator stimulus association, and the third is a comparator stimulus-US association. During testing, the CS evokes two representations of the US. The first is produced directly by the CS-US association. The second is produced indirectly; the CS activates a representation of the comparator stimulus, which in turn activates its own representation of the US. Excitatory responding is predicted when the US representation evoked by the CS is strong relative to the US representation evoked through mediation by the comparator stimuli. Conversely, behavior indicative of conditioned inhibition is predicted when the CS-US association is weak relative to the US representation activated by the comparator stimulus.

One unique successful prediction of the comparator hypothesis is that postconditioning extinction of a CS's training context (i.e., its comparator stimulus) is expected to increase excitatory responding to the CS because of the decreased associative strength of the CS's comparator stimuli relative to that of the CS. Among the findings consistent with these predictions, Matzel, Brown, and Miller (1987) found that posttraining extinction of the training context can attenuate the US-preexposure effect, that is, 
the retarded conditioned responding during training that results from pretraining exposure to the unconditioned stimulus. Also consistent with this view (although open to other interpretations), Kaplan and Hearst (1985) found that posttraining extinction of the training context can enhance responding that was degraded by unsignaled US presentations intermingled among the CS-US training trials. Notably, comparator stimuli have been found to be other cues that were present during training, regardless of where testing takes place (e.g., Kasprow, Schachtman, \& Miller, 1987).

In most research on the comparator hypothesis, the issue of which training context plays the role of comparator stimulus has been clear because the CS has been trained only in one context. Equivalent training of a single CS in two different contexts is a situation in which multiple potential comparator stimuli might compete for associative modulation of responding to the CS. In such a situation, the relative potential of each context to modulate responding to the CS is currently unknown. Presumably, both contexts would become comparator stimuli to some extent, perhaps dependent on the order in which training occurs in the two contexts. In the case in which training in one context precedes training in a second context, posttraining extinction of the first context might enhance responding to the CS more than would extinction of the second context. Such an outcome would indicate a greater comparator role for the first context than for the second context. Conversely, the opposite outcome would suggest a greater comparator role for the second training context. Alternatively, both training contexts might contribute equally to the effective comparator stimulus. In this case, extinction of either context should have the same effect, and extinction of both might be expected to have a greater effect on responding to the CS than extinction of either context alone.

The present experiments investigated potential competition for comparator status between two contexts arising from training a single CS in each context in separate sessions. Training sessions in each context were either sequential or alternated. In both of these contexts, partial reinforcement of the CS $(25 \%)$ was intermingled with a modest density of unsignaled USs (i.e., $\mathrm{CS} \pm /+$ ), thereby providing equivalent contingencies for the $C S$ in each context. The relative importance of the two potential comparator contexts was assessed by posttraining extinction (i.e., deflation) of one or the other training context (Experiment 1) or both training contexts (Experiment 2). To the extent that a training context becomes established as the CS's comparator stimulus, the comparator hypothesis anticipates an increase in responding to the CS as a result of extinction of that context. Hence, the relative influence of extinction of any particular comparator stimulus should be evidenced in the magnitude of the consequent increase in responding to the CS.

\section{EXPERIMENT 1}

Experiment 1 used a $4 \times 3$ factorial design (see Figure 1). Following adaptation to the contexts, the subjects were exposed to one of four training conditions as the first factor. The training conditions were: A-B, in which 6 days of training in Context $A$ were followed by 6 days of training in Context B; ALT, in which training was alternated daily for 12 days between Contexts $A$ and $B$; $\mathrm{HC}-\mathrm{A}$, in which the only training was given in Context $\mathrm{A}$ on the last 6 days of the training phase; and $\mathrm{A}-\mathrm{HC}$, in which the only training was given in Context $A$ on the first 6 days of the training phase. ("HC" refers to homecage exposure during the first and last half of the training phase for Groups HC-A and A-HC, respectively.) The subjects were then given one of three extinction treatments as the second factor: EA, which consisted of extinction of Context A; EB, which consisted of extinction of Context $B$; and NE, in which neither training context was extinguished. For the subjects in Conditions A-HC and $\mathrm{HC}-\mathrm{A}$, for which there was no Context B training, "extinction of Context B" consisted of home-cage exposure. Finally, the subjects were tested for conditioned suppression to the CS in an associatively neutral test context (Context TST) and then for conditioned suppression to the training contexts. Notably, a single test trial was administered to minimize the possibility that the test context would begin to serve as the comparator stimulus for the CS.

If the A-B training condition caused one training context to have a greater influence on CS responding than the other context, then this differential control should be evident in differences in responding to the $\mathrm{CS}$ as a function of whether Context $A$ (initial training context) or Context B (final training context) was extinguished following completion of CS training. Group ALT, for which training was alternated between the two contexts, was expected to show that the two training contexts could become conjoint comparator stimuli. Groups HC-A and $\mathrm{A}-\mathrm{HC}$ were intended to be controls, to determine the effectiveness of Context $A$ as a comparator stimulus when it was the only possible comparator stimulus for the target CS. Additionally, a comparison of responding to the CS between Group A-HC and Group HC-A would provide a basis for determining whether any weakening of Context A's comparator potential in Group A-B relative to Group $\mathrm{HC}-\mathrm{A}$ was due to a difference in the retention interval alone (i.e., forgetting of experience in Context A) or interference as a result of training in Context $B$.

\section{Method}

\section{Subjects}

Forty-eight male and 48 female naive Sprague-Dawley rats, bred in our colony from Holtzman stock (Madison, WI), served as subjects. Weight ranges were $265-405 \mathrm{~g}$ for males and $180-260 \mathrm{~g}$ for 
ADAPTATION

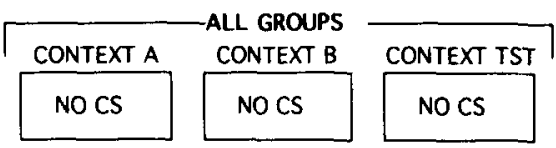

TARGET TRAINING

DAYS 1-6

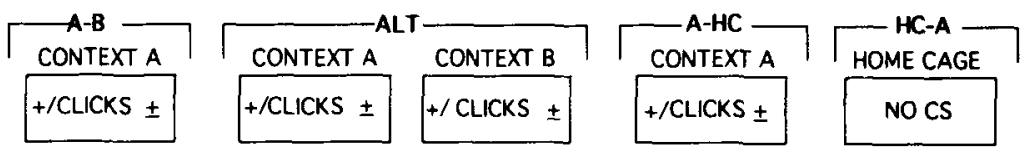

DAYS $7-12$

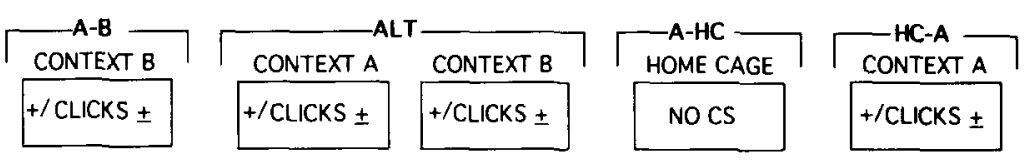

\section{EXTINCTION}

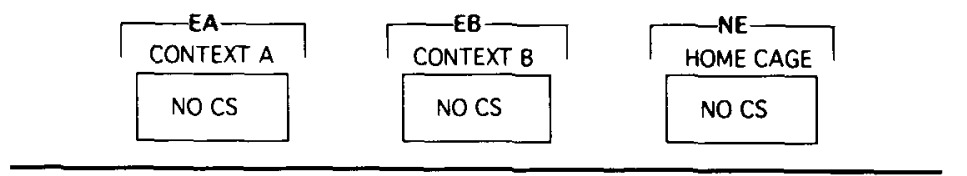

TESTING

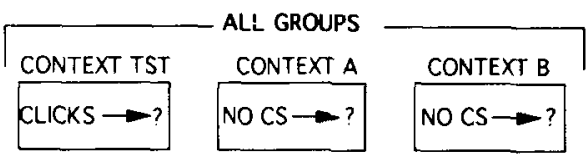

Figure 1. Design of Experiment 1. The experiment was conducted as a 4 (training condition) $\times 3$ (extinction condition) design. Training conditions are represented under the heading "TARGET TRAINING," and extinction conditions are listed under "EXTINCTION." Context A, Context B, and Context TST were three distinct contexts; \pm denotes partial reinforcement of the click CS with footshock, and $+/$ denotes interspersed unsignaled footshock. See text for further details of group treatments.

females. The subjects were individually housed in wire-mesh cages in a vivarium maintained on a 16:8-h light:dark cycle, with experimental sessions conducted approximately midway through the light phase. The subjects were allowed ad-lib access to food (Purina Lab Chow) in their home cages. A progressive water-deprivation schedule was administered over the week prior to the beginning of the experiment, until water availability was limited to $10 \mathrm{~min}$ per day, approximately $1 \mathrm{~h}$ after any scheduled treatment. All the subjects were handled three times weekly for $30 \mathrm{sec}$, from weaning at 22 days of age until the initiation of the study.

\section{Apparatus}

Three types of enclosures were used: $Y, Z$, and Long. Enclosure $Y$ or Enclosure $Z$ served as Context Test (TST), counterbalanced with groups. Enclosure Long served as Context $A$ for half the subjects within each group and as Context B for the remaining subjects in that group. The remaining enclosure served as Context B or Context A, respectively. Enclosure $\mathrm{Y}$ was a clear, Plexiglas chamber in the shape of a box, $22.75 \times 8.25 \times 13.0 \mathrm{~cm}$. The floor was constructed of $0.48-\mathrm{cm}$-diam rods, $1.5 \mathrm{~cm}$ center-to-center, short via NE-2 neons, which allowed a constant-current footshock to be delivered by means of a high-voltage circuit in series with a 1.0-M $\Omega$ resistor. Enclosure $Y$ was not illuminated. Each of six copies of Enclosure $Y$ had its own environmental isolation chest. Background noise (mostly from a ventilation fan) was $74 \mathrm{~dB}(\mathrm{C})$. A 6/sec-click train $8 \mathrm{~dB}(\mathrm{C})$ above background could be presented from a speaker inside the environmental chest. Each chamber was equipped with a water-filled lick tube that extended about $1 \mathrm{~cm}$ from the distal end of a cylindrical niche, the axis of which was perpendicular to the front wall of the enclosure. The niche was $4.5 \mathrm{~cm}$ in diameter, left-right centered with its bottom $1.75 \mathrm{~cm}$ above the floor of the apparatus, and $5.0 \mathrm{~cm}$ deep. There was a photobeam detector $1 \mathrm{~cm}$ in front of the lick tube.

Enclosure $Z$ was a $25.5-\mathrm{cm}-$ long box in a truncated-V shape ( $28 \mathrm{~cm}$ high, $21 \mathrm{~cm}$ wide at the top, $5.25 \mathrm{~cm}$ side at the bottom). Each of six copies of Enclosure $Z$ had its own environmental isolation chest. The floor and sides were constructed of sheet metal. The ceiling was clear Plexiglas. The floor consisted of two parallel metal plates, each $2 \mathrm{~cm}$ wide with a $1.25-\mathrm{cm}$ gap between them. Enclosure $\mathrm{Z}$ was dimly illuminated by a $7.5-\mathrm{W}$ houselight. The 
houselight bulb was mounted on an inside wall of the environmental isolation chest, approximately $30 \mathrm{~cm}$ from the animal enclosure. The light entering the animal enclosure was primarily reflected from the roof of the environmental chest. Background noise (mostly from a ventilation fan) was $74 \mathrm{~dB}(\mathrm{C})$. A 6/sec-click train $8 \mathrm{~dB}(\mathrm{C})$ above background could be presented from a speaker inside the environmental chest. Each chamber was equipped with a water-filled lick tube that extended about $1 \mathrm{~cm}$ into a cylindrical niche, identical to the niche in Enclosure $Y$. There was a photobeam detector $1 \mathrm{~cm}$ in front of the lick tube.

The third type of chamber (of which there were 12 copies) was designated Enclosure Long. These chambers measured $51 \times 16.5 \times$ $75 \mathrm{~cm}$, and had end walls constructed of opaque green Plexiglas and long walls of aluminum. The floor was made of $0.64-\mathrm{cm}$-diam stainless steel rods, $1.8 \mathrm{~cm}$ center-to-center, positioned lengthwise in the chambers and shorted together with $\mathrm{NE}-2 \mathrm{H}$ neon bulbs in series with a $1-\mathrm{M} \Omega$ resistor to produce a constant-current $0.7-\mathrm{mA}$ footshock. This type of chamber was housed in a well-lighted ( $160 \mathrm{~W}$ of fluorescent illumination) experimental room. These chambers were not in environmental isolation chests. A 45- $\Omega, 10-$ cm-square overhead speaker could provide a $6 /$ sec-click train $8 \mathrm{~dB}(\mathrm{C})$ above a 56- $\mathrm{dB}(\mathrm{C})$ background. A lick tube with water was affixed to the end wall of Enclosure Long, but no photocell was present.

\section{Procedure}

Acclimation to Context TST (Day 1). All the subjects were placed in their test context (Enclosure Y or Enclosure Z, counterbalanced within groups) for a 60-min acclimation session.

Acclimation to Contexts A and B (Day 2). During the first session of the day, the subjects were placed in Context A or Context B for $10 \mathrm{~min}$ with water available. For half of the animals in each treatment group, Context A was Enclosure Long, and for the remaining animals it was either Enclosure $Y$ or Enclosure $Z$, whichever had not been designated Context TST. The order of exposure to the two contexts was counterbalanced within groups. The half of the subjects in Conditions A-HC and HC-A that were scheduled for adaptation first to Context $B$ (which was the home cage for these animals) received access to water in the home cage for the first acclimation session of Day 2. After this first session, all the subjects were placed in (or remained in) the home cage for $1 \mathrm{~h}$ without water, and then spent an additional $10 \mathrm{~min}$ in Context B or Context $A$ (whichever context had not been used during the first session). The subjects in Conditions $\mathrm{A}-\mathrm{HC}$ and $\mathrm{HC}-\mathrm{A}$ that were placed in Context $A$ during the first session of Day 2 were given equivalent access to water in the home cage (i.e., their Context B). Latencies to complete the 1st and 2nd 5 cumulative seconds of licking were recorded in all enclosures except Enclosure Long and the home cage, which lacked photobeam detectors. The subjects were subsequently assigned to 1 of 12 groups (counterbalanced for sex) for the remaining two phases of the experiment (all $n s=8$ ).

Training (Days 3-14). Training, when it was given, was the same for all the subjects, regardless of the context in which it was given. It consisted of eight presentations per session of the 30-sec click $\mathrm{CS}$, two of which terminated with the onset of a $0.5-\mathrm{sec}$ footshock US. The subjects were also presented with 32 unsignaled USs during each daily 60 -min training session. The intertrial intervals were varied pseudorandomly within sessions, with none longer than $11 \mathrm{~min}$ nor shorter than $3 \mathrm{~min}$. No unsignaled US occurred within $60 \mathrm{sec}$ of any CS. In order to reduce the likelihood of the subjects' learning the temporal patterning of trials, two different sequences of trials were used in pseudorandom order across sessions for each treatment group. Groups A-B/EA, A-B/EB, and A-B/NE received training in Context $A$ during the first 6 days of this phase, and training in Context B during the second 6 days. For Groups ALT/EA, ALT/EB, and ALT/NE, training was alternated daily between Contexts $\mathrm{A}$ and $\mathrm{B}$. Consequently, these groups received six sessions in each context over the 12 days of training, starting with Context $A$ and ending with Context B. Groups A-HC/EA, A-HC/EB, and $\mathrm{A}-\mathrm{HC} / \mathrm{NE}$ were trained in Context $\mathrm{A}$ on the first 6 days and then spent the remainder of this phase in the home cage. Groups $\mathrm{HC}$ $\mathrm{A} / \mathrm{EA}, \mathrm{HC}-\mathrm{A} / \mathrm{EB}$, and $\mathrm{HC}-\mathrm{A} / \mathrm{NE}$ remained in the home cage for the first 6 days of this phase and then were trained in Context $A$ during the second 6 days of this phase.

Context extinction (Days 15-22). During 60-min daily sessions, the subjects in Groups A-B/EA, ALT/EA, A-HC/EA, and HC-A/EA received exposure to Context $A$ without any discrete CSs or USs being presented. The subjects in Groups A-B/EB, ALT/EB, $\mathrm{A}-\mathrm{HC} / \mathrm{EB}$, and $\mathrm{HC}-\mathrm{A} / \mathrm{EB}$ received equivalent exposure to Context $B$ (which was the home cage for the latter two groups). Times to complete the first and second 5 cumulative lick seconds were recorded for these subjects, except when the sessions occurred in Enclosure Long or the home cage. Groups A-B/NE, ALT/NE, $\mathrm{A}-\mathrm{HC} / \mathrm{NE}$, and $\mathrm{HC}-\mathrm{A} / \mathrm{NE}$ stayed in the home cage with water present during these $60-\mathrm{min}$ sessions.

Reshaping (Days 23-25). In order to restabilize baseline drinking rates after training, the subjects received 60 -min daily sessions in Context TST. Times to complete the first and second 5 cumulative seconds of drinking were recorded. Any subject failing to complete 5 cumulative seconds of licking within the first $60 \mathrm{sec}$ of the Day 25 reshaping session was eliminated from the study. No animals in Experiment 1 met this criterion.

CS test in Context TST (Day 26). All the subjects were placed in Context TST for an 11-min session. When a subject completed 5 cumulative seconds of licking, the click CS was presented and remained on for the remainder of the session. Times to complete the first 5 cumulative seconds of drinking, in the presence of the context alone, and the second 5 cumulative seconds of drinking, in the presence of the click train, were recorded. This latter latency, time to complete 5 cumulative seconds of drinking in the presence of the CS, constituted our primary dependent variable. Rats that did not complete the second 5 cumulative seconds of drinking within $10 \mathrm{~min}$ were assigned scores of $600 \mathrm{sec}$. This applied to 2 subjects in Group HC-A/EA and 1 subject in Group A-B/EB. Rats that did not complete the first 5 cumulative lick seconds within $60 \mathrm{sec}$ were eliminated from the study. This applied to 1 subject in Group A-HC/EA and 1 subject in Group HC-A/NE.

Test in Context A or Context B (Day 27). The subjects that had received CS training only in Context $\mathbf{A}$ were tested for lick suppression to the background cues of that context when that context corresponded to Enclosure $Y$ or Enclosure Z. (Enclosure Long lacked equipment to record lick suppression.) Groups that had received CS training in both Context $A$ and Context $B$ were divided equally, with half the subjects tested in Context $A$ and half tested in Context B. Assignment to subgroups for the purpose of this test was random, except that no subjects were assigned to a context corresponding to Enclosure Long because of the absence there of a photobeam to allow collection of lick data. During an 11-min session, times to complete 5 cumulative seconds of drinking were recorded.

\section{Results and Discussion}

Results indicated that in the A-HC and $\mathrm{HC}-\mathrm{A}$ training conditions, posttraining extinction of Context $A$ enhanced responding to the CS. Following A-B training, responding to the CS was enhanced by posttraining extinction of Context $B$ but not Context A. In the ALT training condition, extinction of neither Context A nor Context B influenced responding to the CS. Details and analyses supporting these conclusions are explicated below.

A log (base 10) transformation was performed on all latency scores to improve the normality of the data, 
thereby permitting the use of parametric statistics. No difference between groups in times to complete the first or second 5 cumulative seconds of drinking was found on either the adaptation day or the last baseline recovery day, nor was there a difference in times to complete the first 5 cumulative seconds of licking on Day 26 (i.e., just prior to the presentation of the test $\mathrm{CS}$ ). One subject in Group A-B/EA died prior to testing.

Essential to the interpretation of CS responding was determination of whether responding to the context was affected by the context extinction manipulation. To this end, scores from the training context(s) tests on Day 27 were analyzed in two separate analyses of variance (ANOVAs). The subjects in Conditions A-HC and HC-A were tested only in Context A because these subjects had no Context B. Moreover, data were obtained only when Context A was Enclosure $\mathrm{Y}$ or Enclosure $\mathrm{Z}$ but not when Context $\mathrm{A}$ was Enclosure Long because Enclosure Long lacked a photobeam to assess licking. The Day 27 data for these subjects were analyzed in a 2 (training: A-HC or HC-A) $\times$ 2 (extinction: EA or NE [including EB]) ANOVA. As expected, the only significant finding was a main effect of extinction $[F(1,20)=3.57, p<.05]$. The sizes of the Day 27 subgroups were too small $(n s=4$ or 8 ) for meaningful planned comparisons between individual groups. But the main effect of extinction arose from the subjects that had received extinction of Context $A$ following training (i.e., Groups A-HC/EA and HC-A/EA; mean latency to complete the first 5 cumulative seconds of licking $=1.15 \mathrm{log} \mathrm{sec}$ ) who displayed less fear of Context $A$ than the subjects that had only exposure to the home cage following training (i.e., Groups $\mathrm{A}-\mathrm{HC} / \mathrm{NE}$ and $\mathrm{HC}-\mathrm{A} / \mathrm{NE}$; mean latency to complete the first 5 cumulative seconds of licking $=1.89 \log \mathrm{sec}$ ). There was no significant main effect of training and no extinction $x$ training interaction.

The second ANOVA of Day 27 data was performed on scores from the subjects in Conditions A-B and ALT. Their data were analyzed with a 2 (training: A-B or ALT) $\times 3$ (extinction: EA, EB, or NE) $\times 2$ (Day 27 test context: A or B) ANOVA. The only significant finding was a main effect of extinction $[F(2,36)=16.61, p<.001]$. No planned comparisons were performed due to the small sizes of the subgroups $(n s=4)$, which yielded low statistical power. However, the subjects tested in training contexts in which posttraining exposure had not occurred tended to exhibit more fear of the context (mean latency to complete the first 5 cumulative seconds of licking = $1.70 \mathrm{log} \mathrm{sec}$ ) than the subjects tested in contexts to which they had subsequently been exposed (i.e., extinguished; mean latency to complete the first 5 cumulative seconds of licking $=1.04 \log \mathrm{sec}$ ). This outcome was again consistent with the expectation that subjects would have lower latencies when tested in a context to which they were exposed (i.e., extinguished) following training. Moreover, it testifies to the animals' having discriminated between the two training contexts.

Most important to the present experiment are the data from the CS test on Day 26. Mean suppression scores in the presence of the clicks on Day 26 are depicted in Figure 2. A $4 \times 3$ ANOVA detected a main effect of training $[F(3,81)=8.00, p<.001]$, as well as a main effect of the extinction manipulation $[F(2,81)=10.58, p<.001]$. More important, there was a training $\times$ extinction interaction $[F(6,81)=15.39, p<.001]$. Planned comparisons among groups trained only in one context revealed that Group A-HC/EA suppressed more to the clicks than did Group A-HC/EB or Group A-HC/NE $[F s(1,81) \geq$ 18.93, ps <.001]. Similarly, Group HC-A/EA suppressed more than either Group HC-A/EB or Group HC-A/NE $\left[F_{s}(1,81) \geq 22.70\right.$, ps $\left.<.001\right]$. Thus, for the animals trained only in Context $A$, posttraining extinction of Context $A$ resulted in increased responding to the click CS, suggesting that for these animals, Context A had become the comparator stimulus for the target CS.

The preceding analysis of conditioned suppression to the clicks assumed that a 4 (training: A-B, A-HC, HC-A, or ALT) $\times 3$ (extinction: EA, EB, or NE) design was used. However, for Conditions A-HC and HC-A, Context B (in which no CS or US was scheduled to occur) was the home cage (i.e., HC). Consequently, Groups $\mathrm{A}-\mathrm{HC} / \mathrm{EB}$ and $\mathrm{A}-\mathrm{HC} / \mathrm{NE}$ were identically treated and Groups $\mathrm{HC}-\mathrm{A} / \mathrm{EB}$ and $\mathrm{HC}-\mathrm{A} / \mathrm{NE}$ were identically treated. Although there is no obvious reason to expect that regarding these identically treated groups as separate groups could have artificially produced the differences observed in the above analysis, we reanalyzed the data collapsing across identically treated groups. That is, the animals in Group A-HC/EB were added to Group

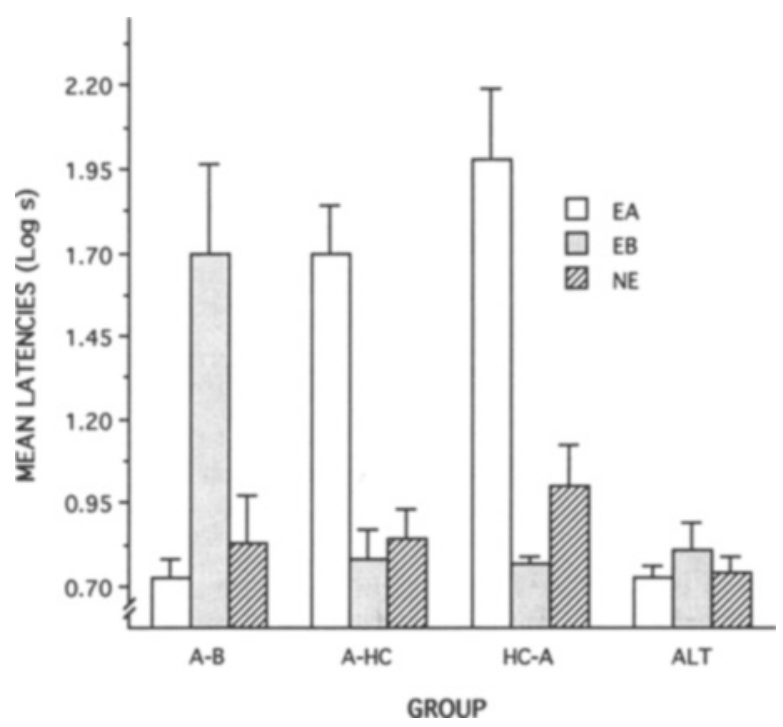

Figure 2. Results of Experiment 1. Mean latencies in Context TST to complete 5 cumulative seconds of drinking in the presence of the click CS. The CS was trained first in Context $A$ and then in Context B (A-B), in Context A only (A-HC and HC-A), or in Contexts A and B alternately (ALT). Then subjects received extinction of Context A (EA), extinction of Context B (EB), or no extinction treatment (NE). See text for details of group trentments. Brackets denote standard errors. 
A-HC/NE and the animals from Group HC-A/EB were added to Group HC-A/NE. Two ANOVAs were then performed: a 2 (training: A-B or ALT) $\times 3$ (extinction: EA, EB, or NE) ANOVA, and a 4 (training: A-B, A-HC, HC-A, or ALT) $\times 2$ (extinction: EA or NE) ANOVA. The $2 \times 3$ ANOVA detected main effects of both training $[F(1,41)=10.10, p<.01]$ and extinction $[F(2,41)=$ $9.01, p<.001]$, as well as a training $\times$ extinction interaction $[F(2,41)=6.87, p<.01]$, all of which arose from the high suppression scores of Group A-B/EB relative to the other five groups in this analysis. The $4 \times 2$ ANOVA also detected main effects of both training $[F(3,68)=20.75, p<.001]$ and extinction $[F(1,68)=$ $16.31, p<.001]$, as well as a training $\times$ extinction interaction $[F(3,68)=10.43, p<.001]$, all of which arose from the high suppression scores of Groups A-HC/EA and $\mathrm{HC}-\mathrm{A} / \mathrm{EA}$ relative to the other six groups in this analysis. Thus, the subjects that had been trained in a single training context and subsequently had had that training context extinguished displayed greater fear of the clicks than did the subjects that were identically trained but that had received either no posttraining extinction of either context or posttraining extinction of the other "training" context (in which click training had not occurred). This demonstrates the basic effect predicted by the comparator hypothesis that extinction of a CS's training context can increase the response potential of that CS.

The role of multiple training contexts as comparator stimuli was investigated in Conditions A-B and ALT. For Condition A-B, only extinction of Context B (i.e., EB) had any effect on suppression to the clicks relative to no context extinction (i.e., NE) $[F(1,81)=21.71, p<$ .001]. (Group A-B/EA actually exhibited a nonsignificant tendency toward shorter latencies than Group A-B/NE.) For Condition ALT, neither extinction of Context A nor extinction of Context B resulted in more fear of the clicks than did no context extinction (all $F \mathrm{~s}<1$ ).

For Condition A-B, Context B claimed more of a comparator role than Context $A$. This is evident from the observation that posttraining extinction of Context B increased the fear response to the click $C S$ relative to the no-context extinction control (i.e., Group A-B/NE), whereas extinction of Context $\mathrm{A}$ did not. This finding is consistent with the most recent training experience determining the outcome of the competition between Contexts $A$ and $B$ for the role of effective comparator stimulus.

In Condition ALT, alternation of the training between Contexts A and B prevented either context from being notably the more recent at the time of testing, in contrast with Condition A-B. Consequently, ALT treatment might have been expected to produce a situation in which both Context A and Context B were, to some extent, effective comparator stimuli. Thus, it was surprising in Condition ALT that extinction of neither Context A nor Context $B$ augmented responding to the clicks, even though the context extinction manipulation was effective in in- creasing responding to the $\mathrm{CS}$ in the other training conditions (i.e., $\mathrm{A}-\mathrm{HC}, \mathrm{HC}-\mathrm{A}$, and $\mathrm{A}-\mathrm{B}$ ).

There are several potential explanations for this outcome. First, neither of the contexts may have become comparator stimuli as a result of ALT treatment. However, this is not a particularly attractive view in light of the finding that at least one of the contexts functioned as a comparator stimulus under somewhat similar conditions (i.e., Condition A-B). Second, both contexts may have become comparator stimuli for the CS in some way that did not afford an increase in responding to the clicks as a consequence of extinguishing either context alone. However, the hypothesis that the effective comparator stimulus for the CS was an average of the potential comparator roles of each training context separately is inadequate, because this view would anticipate some enhancement of responding to the $\mathrm{CS}$ as a result of extinguishing either context, an outcome that was not observed. Alternatively, an algebraic summation of the comparator status of the two training contexts could have left the CS with a very low response potential, yielding a floor effect, even after extinction of one training context. Because the comparator role of one context alone was sufficient to largely attenuate responding to the $\mathrm{CS}$ after training in that context (i.e., Conditions $\mathrm{A}-\mathrm{HC}$ and $\mathrm{HC}-\mathrm{A}$ ), the two training contexts of Condition ALT may have summated their comparator status to yield a "super-low" response potential for the CS, which did not rise above behavioral threshold when only one training context was subsequently extinguished. Thus, leaving either of the two context-US associations intact may have sufficed to attenuate responding to the CS.

\section{EXPERIMENT 2}

In Experiment 1, no increase in conditioned responding to the click $C S$ as a result of extinguishing either Context $A$ or Context $B$ was observed in the subjects that received repeated cycles of training in Context $A$ and $C$ ntext B (i.e., Condition ALT). Consequently, it was unclear after ALT training whether neither training context served as the comparator stimulus for the CS or whether the two training contexts summated in serving as comparator stimuli for the CS. Experiment 2 examined this issue by extinguishing both Context $\mathrm{A}$ and Context $\mathrm{B}$ following ALT training.

\section{Method}

\section{Subjects}

Twenty-four male and 24 female naive Sprague-Dawley rats, bred in our colony from Holtzman stock (Madison, WI), served as subjects. Weight ranges were 270-395 $\mathrm{g}$ for males and 170-260 $\mathrm{g}$ for females. The subjects were maintained in the same manner as those in Experiment 1.

\section{Apparatus}

Contexts TST, A, and B consisted of the same enclosures (Y, Z, and Long) used in Experiment 1. Again, because of Enclosure Long's lack of a photobeam to assess licking, it never served 
as Context TST. Enclosures $\mathrm{Y}$ and $\mathrm{Z}$ were counterbalanced in serving as Context TST.

\section{Procedure}

Adaptation and training (Days 1-14). Acclimation of all the subjects to Contexts TST, A, and B occurred on Days 1 and 2 in the same manner as in Condition ALT in Experiment 1. Training for all the subjects on Days 3-14 was also the same as that for Condition ALT in Experiment 1. That is, sessions in which two of eight clicks were reinforced and 32 unsignaled USs were presented occurred alternately in Contexts A and B on successive days.

Context extinction (Days 15-22). During 60-min daily sessions, the subjects in Group EA received exposure to Context A with no discrete CSs or USs presented. The subjects in Group EB received equivalent exposure to Context B. Group EAB subjects received two daily 60 -min extinction sessions, one in Context $A$ followed by one in Context B, separated by $4 \mathrm{~h}$ in the home cage. Times to complete the first and second 5 cumulative lick seconds were recorded for these subjects, except when the sessions occurred in Enclosure Long. Group NE stayed in the home cage during this phase of treatment, receiving access to water equivalent to that for Group EAB. Additionally, Groups EA and EB received water in the home cage to match the water access of Group EAB.

Reshaping (Days 23-25). In order to restabilize baseline drinking rates after training and context extinction, the subjects received 60-min daily sessions in Context TST. Times to complete the first and second 5 cumulative seconds of drinking were recorded.

Testing (Days 26-27). Testing of the associative status of the target CS on Day 26 and the training contexts on Day 27 was performed in the same manner as in Experiment 1. Lick suppression in the presence of the CS was assessed on Day 26 in Context TST. On Day 27, during the training context test, the subjects were tested in whichever context (A or B) was either Enclosure Y or Enclosure $Z$, due to the absence of a photobeam circuit in Enclosure Long. Thus, half the subjects in each group were tested in Context $\mathbf{A}$ and the other half were tested in Context $\mathbf{B}$.

\section{Results and Discussion}

Results indicated that extinguishing both training contexts, but not either context alone, was effective at increasing conditioned responding to the CS. This indicates that following ALT training, both training contexts became comparator stimuli for the target CS. Details and analyses supporting these conclusions are presented below.

Two subjects were eliminated from all statistical analyses for failure to complete their first 5 cumulative lick seconds within $60 \mathrm{sec}$ on the last day of lick baseline restabilization (i.e., Day 25). No difference between groups in times to complete the first or second 5 cumulative seconds of drinking was found on either the adaptation day or the last baseline recovery day, nor did the groups differ in time to complete the first 5 cumulative seconds of licking on Day 26 (i.e., just prior to the presentation of the test CS).

Interpretation of CS data required ensuring that the context extinction manipulation effectively reduced fear of the training context(s). A 4 (extinction condition: EA, EB, EAB, or NE) $\times 2$ (training context tested: Context $A$ or Context B) ANOVA was performed on the training context data on Day 27. This revealed a main effect of extinction $[F(3,38)=16.92, p<.001]$ and a strong tendency toward an interaction of extinction treatment and context tested $[F(3,38)=2.72, p<.06]$, which appar- ently arose from less suppression by the subjects tested in an extinguished context than by the subjects tested in a nonextinguished context. Group sizes ( $n s$ of 5 and 6) were too small to allow sufficient statistical power for planned comparisons. However, all tendencies were consistent with posttraining exposure to a training context's reducing fear of that context (i.e., extinction). Mean latency to complete 5 cumulative seconds of licking in an extinguished context was $1.23 \log \mathrm{sec}$, whereas in a nonextinguished context it was $2.18 \mathrm{log} \mathrm{sec}$.

Most important was testing of the clicks on Day 26 in Context Test (see Figure 3), which was analyzed with a 2 (extinction of Context A: yes or no) $\times 2$ (extinction of Context B: yes or no) ANOVA. This analysis revealed main effects of Context A extinction $[F(1,42)=11.18$, $p<.01]$ and Context $B$ extinction $[F(1,42)=18.60$, $p<.001]$, as well as an interaction of the two extinction conditions $[F(1,42)=13.45, p<.001]$. Planned comparisons indicated that Group EAB showed greater suppression to the clicks than any of the other groups $[F(1,42)=25.82, p<.001]$, whereas neither Group EA nor Group EB differed from Group NE (both $F \mathrm{~s}<1$ ). Thus, only extinction of both training contexts was effective at increasing the magnitude of the conditioned response to the clicks.

\section{GENERAL DISCUSSION}

Following partially reinforced CS trials that were intermingled with unsignaled USs, extinction of the training context enhanced responding to the CS under certain circumstances. Specifically, the animals trained in a single

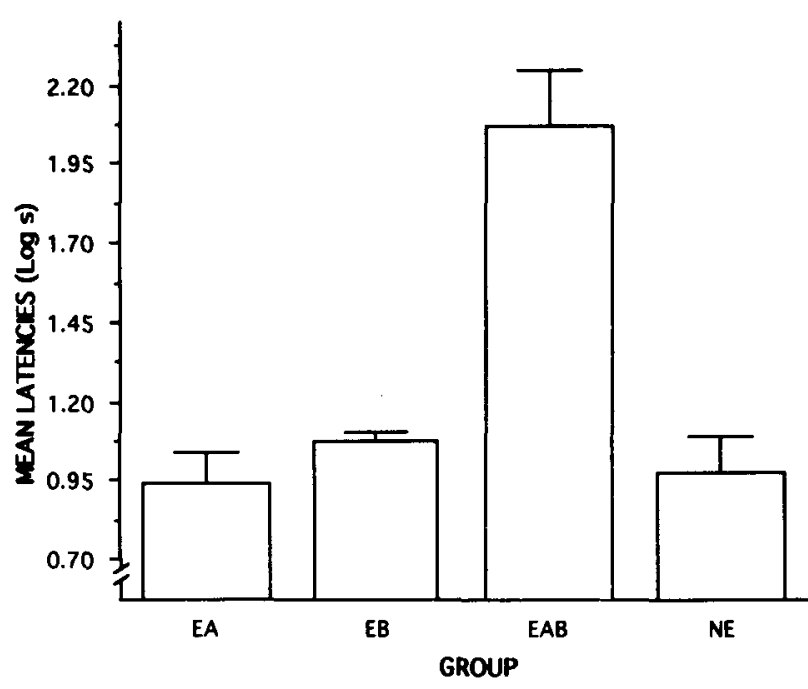

Figure 3. Results of Experiment 2. Mean latencies in Context TST to complete 5 cumulative seconds of drinking in the presence of the click CS. All groups were trained alternately in Contexts A and B, identical to Condition ALT in Experiment 1. Then subjects received extinction of Context A (EA), extinction of Context B (EB), extinction of both Contexts $A$ and $B$ (EAB), or no extinction treatment (NE). Brackets denote standard errors. 
context exhibited heightened responding to the CS when that training context was extinguished in Experiment 1 . Furthermore, the animals for which training of the CS occurred first in one context and then in a second context displayed enhanced fear only as a result of extinguishing the second context; that is, recency determined the effective comparator stimulus for the CS. The animals trained in the two contexts on alternating sessions exhibited no increment in responding to the CS as a result of extinction of only one of the contexts, but when both training contexts were extinguished in Experiment 2, enhanced responding to the CS was evident.

The observed increases in responding to a CS as a consequence of extinguishing other cues that were present during training of the CS are consistent with the comparator hypothesis (e.g., Miller \& Matzel, 1988), which posits that responding to a CS is directly related to the strength of the CS-US association and is inversely related to the associative strength of the background cues (i.e., comparator stimuli) that were present during training. Superficially, these data are also consistent with Gordon's event reactivation data (Gordon, Smith, \& Katz, 1979). Specifically, Gordon's framework might suggest that posttraining exposure to the context would reactivate the memory for CS-US pairings, and thereby enhance responding to the CS. However, in an avoidance procedure, it was found that longer periods of exposure resulted in response decrement rather than enhancement. Therefore, it is unlikely that the present extended context exposure would enhance target CS responding through the mechanisms discussed by Gordon and his colleagues.

Support for the basic comparator effect of context extinction on CS responding is most straightforward in Conditions $\mathrm{A}-\mathrm{HC}$ and $\mathrm{HC}-\mathrm{A}$ from Experiment 1, which were uncomplicated by CS training in Context $\mathrm{B}$. In both of these conditions, responding to the CS was markedly enhanced by extinction of the training context (i.e., Context $\mathrm{A}$ ) relative to the no-extinction condition. The fact that increased responding to the $\mathrm{CS}$ was observed in both Condition A-HC and Condition HC-A as a result of extinction of the training context also indicates that extinction of the comparator stimulus does not have to occur soon after CS training in order to produce facilitated responding to the $\mathrm{CS}$. In fact, there was almost no difference between Conditions $\mathrm{A}-\mathrm{HC}$ and $\mathrm{HC}-\mathrm{A}$ in the magnitude of enhanced responding to the $C S$ as a result of extinction of Context A (see Figure 2), suggesting that the 6-day difference in intervals between training and extinction treatment did not undermine either the CS-comparator association or the comparator-US association.

A novel finding in Experiment 1 is that Context $B$ alone appeared to serve as the effective comparator stimulus for the CS in animals that were first trained with the CS in Context A and then trained with the CS in Context B (i.e., Condition A-B). Two lines of evidence support this conclusion. First, posttraining extinction of Context B in A-B subjects appeared to largely restore responding to the CS. That is, responding to the CS was similar to that of sub- jects in Groups A-HC/EA and HC-A/EA, which had had their sole training context extinguished. This demonstrates that Context $B$ was the major comparator stimulus for A-B animals. Second, extinction of Context A following A-B training had no apparent effect on responding to the CS. That is, responding to the CS was similar to that of subjects in Group A-B/NE, which had no context extinguished after CS training. Collectively, these observations indicate that only Context $B$ was an effective comparator stimulus for the CS following A-B training. The observed effect of extinguishing Context $A$ on responding to the CS in Condition A-HC implies that Context $\mathrm{A}$ was also the CS's comparator stimulus for Condition A-B after training in Context $A$ and prior to training in Context $B$. Thus, training in Context $B$ after completion of training in Context $\mathrm{A}$ apparently resulted in Context $\mathrm{B}$ displacing, or effectively competing with, Context $\mathrm{A}$ as the comparator stimulus for the CS.

One possible explanation for the displacement as a comparator stimulus of Context A by Context B in A-B subjects is that training in Context $B$ may have retroactively interfered with the comparator role of Context A. In principle, training in Context $B$ may have interfered with either the CS-Context A association or the Context A-US association. According to the comparator hypothesis, degradation of either of these associations to Context $A$ would have attenuated the potential of Context $\mathrm{A}$ to modulate responding to the CS. However, the considerable fear of Context A displayed on Day 27 by subjects in Group $\mathrm{A}-\mathrm{B} / \mathrm{EB}$ is indicative of a strong Context A-US association, which argues against the possibility that the Context A-US association was degraded by subsequent training in Context B. Therefore, a retroactive interference account of the present results must assume that a CS-Context $B$ association acquired during training in Context $B$ interfered with the CS-Context $A$ association, thereby reducing the potential of Context $A$ to modulate responding to the CS.

The greater consequences after A-B training of extinguishing Context $\mathrm{B}$ than of Context $\mathrm{A}$ on $\mathrm{CS}$ responding are clearly inconsistent with the initial training context predominating as the comparator stimulus. Similarly, the present results from the A-B animals are inconsistent, with both potential comparator stimuli being rendered effective as comparator stimuli by sequential training. This latter hypothesis is not tenable because it cannot explain why Context B was the sole comparator stimulus in Condition A-B. If both contexts contributed equally to the effective comparator stimulus for the CS, then extinguishing one context would not be expected to have a larger effect on conditioned responding than extinguishing the other context.

An alternative to the retroactive interference explanation of the greater incremental effect of posttraining extinction of Context B than Context A in A-B subjects can be found in variable salience. Specifically, Context B may have become more salient than Context A during the second half of training, and hence may have been the 
predominant comparator stimulus at the time of extinction and/or testing. Although this view is similar to that based on retroactive interference, in principle it could be differentiated from the retroactive interference explanation by variation of the retention interval. The saliency explanation predicts no loss of Context B's predominance as the CS's comparator stimulus with increasing retention interval, whereas retroactive interference ordinarily wanes with increasing retention intervals. Thus, differences in the effects of posttraining extinction of Contexts $A$ and $B$ would also be expected to wane with increasing retention intervals. Because the present research did not systematically vary the training-to-test retention interval for Condition A-B, both the saliency and retroactive interference explanations remain plausible.

An additional finding of note is that in Experiment 2, following alternating training in two different contexts (i.e., Condition ALT), both contexts appeared to serve as the comparator stimulus for the CS, whereas following serial training in two contexts (i.e., Condition A-B from Experiment 1), the second context appeared to act as the sole comparator stimulus. Alternating training context in Experiment 2 seemingly caused the comparator potential of both training contexts to contribute to the effective comparator stimulus, such that, to impair responding to the target CS, it was sufficient for either context alone to remain excitatory. Presumably, extinction of either context alone was ineffective due to a floor effect in responding to the CS.

Sharing of the comparator role by two cues was seen recently in our laboratory when comparator cues were punctate CSs rather than contexts (Barnet, Grahame, \& Miller, 1992). In that experiment, pairings of a target CS with a US were immediately preceded by a second CS and followed by a third CS. This allowed both the preceding and the following CSs to become comparator stimuli. Responding to the target CS was seen only when both the preceding and the following CSs were extinguished following training, but not when only one of the comparator CSs was extinguished. The Barnet et al. results, although from a quite different training situation than the present series, are congruent with the present results in that they suggest that when two comparator cues are established at approximately the same time, extinction of one of the comparator cues is insufficient to produce enhanced responding to the target CS.

Despite the apparent congruence between the present results and data from prior comparator experiments, alternative, noncomparator explanations of the current observations can be formulated. An anonymous reviewer noted that, without posttraining extinction of the comparator stimulus, generalization between the test context and the training context might have permitted the test context to prime the representations of the CS and the US at the beginning of the CS test session. This would be expected to attenuate conditioned responding to the CS when the CS was presented during testing. Moreover, posttraining extinction of the training context (i.e., the comparator stimulus) would be expected to weaken this priming effect, thereby strengthening conditioned responding to the CS during testing. This view cannot be categorically rejected based on the present data. However, the present results include evidence for strong differentiation among the two training contexts and the test context, which were counterbalanced. Good differentiation among the contexts would be expected to minimize generalization. Moreover, other successful comparator studies have used discrete comparator stimuli (e.g., Hallam et al., 1990), which were even less likely to generalize to the test context used in those studies. Hence, their outcomes do not lend themselves to such an explanation. For both of these reasons, we are inclined to favor comparator interpretations of the present data.

One paradox of comparator phenomena is why responding to the target CS is inversely related to the associative strength of the comparator stimuli rather than directly enhanced by that associative strength. If the CS is associated with the comparator stimulus and the comparator stimulus is associated with the US, as is assumed by the comparator hypothesis, the CS might be expected to accrue added excitatory strength from this second-order linkage to the US. We cannot resolve this paradox based on existing data. However, we suspect on empirical grounds that when most of the CS-comparator stimulus pairings are clustered among themselves and removed from the comparator stimulus-US pairings, the CS is apt to accrue second-order associative strength from the CS-comparator stimulus association. In contrast, when these two types of trials are interspersed, comparator effects are more apt to emerge. This is exemplified by second-order conditioning and Pavlovian conditioned inhibition, the latter of which we view as a comparator phenomenon (e.g., Hallam et al., 1990). The procedures for producing secondorder conditioning and Pavlovian conditioned inhibition are highly similar, but their behavioral outcomes are nearly opposite. Conventional procedures for producing second-order conditioning have all the pairings of the firstorder excitor and the US occurring prior to any pairings of the target CS and the first-order excitor. In contrast, conventional procedures for producing conditioned inhibition have the pairings of the first-order excitor and the US interspersed among the nonreinforced pairings of the target CS (i.e., the conditioned inhibitor) and the firstorder excitor. In the present research, we attempted to bias the training contexts toward yielding comparator effects by interspersing unsignaled US presentations among the CS training trials. Whether or not differences in distribution of the two types of trials are really the basis for differentiating between response-enhancing and responsediminishing effects of within-compound associations will require more research.

The present data widen the applicability of the comparator hypothesis to CSs trained in more than one context by suggesting that the context from among multiple training contexts that acquires the capacity to modulate responding to a CS depends on the nature of CS training 
in those contexts (e.g., sequential vs. alternated). Additionally, the results are congruent with other demonstrations in our laboratory that stimuli can compete to serve as the comparator stimulus for a particular CS (Miller, Esposito, \& Grahame, 1992). The conditions, other than trial order, that might favor displacement of one comparator stimulus by another have yet to be determined, but number of training trials in each context is a likely variable for future investigation.

\section{REFERENCES}

Barnet, R. C., Grahame, N. J., \& Miller, R. R. (1992). Local time horizons in Pavlovian learning. Manuscript submitted for publication. Gordon, W. C., Smith, G. J., \& Katz, D. S. (1979). Dual effects of response blocking following avoidance learning. Behavior Research \& Therapy, 17, 479-487.

Hallam, S. C., Matzel, L. D., Sloat, J. S., \& Miller, R. R. (1990). Excitation and inhibition as a function of posttraining extinction of the excitatory cue used in Pavlovian inhibition training. Learning \& Motivation, 21, 59-84.

Kaplan, P. S., \& Hearst, E. (1985). Excitation, inhibition, and context: Studies of extinction and reinstatement. In P. D. Balsam \& A. Tomie (Eds.), Context and learning (pp. 195-224). Hillsdale, NJ: Erlbaum.

Kasprow, W. J., Schachtman, T. R., \& Miller, R. R. (1987). The comparator hypothesis of conditioned response generation: Manifest conditioned excitation and inhibition as a function of relative excit- atory associative strengths of $\mathrm{CS}$ and conditioning context at the time of testing. Journal of Experimental Psychology: Animal Behavior Processes, 13, 395-406.

Matzel, L. D., Brown, A. M., \& Miller, R. R. (1987). Associative effects of US preexposure: Modulation conditioned responding by an excitatory training context. Journal of Experimental Psychology: Animal Behavior Processes, 13, 65-72.

Miller, R. R., Barnet, R. C., \& Grahame, N. J. (1992). Responding to a conditioned stimulus depends on the current associative status of other cues that were present during training of that specific stimulus. Journal of Experimental Psychology: Animal Behavior Processes, 18, 251-264.

Miller, R. R., Esposito, J. J., \& Grahame, N. J. (1992). Overshadowing-like effects between potential comparator stimuli: Covariation in comparator roles of context and punctate excitor used in inhibitory training as a function of excitor salience. Learning \& Motivation, 23, 1-26.

Miller, R. R., Hallam, S. C., \& Grahame, N. J. (1990). Inflation of comparator stimuli following CS training. Animal Learning \& Behavior, 18, 434-443.

MilleR, R. R., \& MATZEL, L. D. (1988). The comparator hypothesis: A response rule for the expression of associations. In $\mathbf{G}$. H. Bower (Ed.), The psychology of learning and motivation (Vol. 22, pp. 51-92). Orlando, FL: Academic Press.

Miller, R. R., \& Schachtman, T. R. (1985). The several roles of context at the time of retrieval. In P. D. Balsam \& A. Tomie (Eds:), Context and learning (pp. 167-194). Hillsdale, NJ: Erlbaum.

(Manuscript received November 25, 1991; revision accepted for publication April 9, 1992.) 